\title{
Análisis cristalográfico, morfológico, eléctrico, óptico y magnético del nuevo material $\mathrm{Dy}_{2} \mathrm{BiFeO}_{6}$
}

\section{Crystallographic, morphologic, electric, optical and magnetic analysis of the $\mathrm{Dy}_{2} \mathrm{BiFeO}_{6}$ novel material}

\author{
K.Y. Bustos Garnica \\ R. Cardona ${ }^{\mathrm{a}}$ \\ D.A. Landínez Télleza \\ C.A. Parra Vargas ${ }^{b}$ \\ J. Roa-Rojasa ${ }^{*}$
}

Fecha de Recepción: 06 - abr. - 2017

Fecha de Aceptación: 29 - ago. - 2017

\section{Resumen}

En este trabajo reportamos el análisis estructural, morfología superficial, ordenamiento magnético, respuesta dieléctrica y característica óptica de la nueva perovskita compleja $\mathrm{Dy}_{2} \mathrm{BiFeO}_{6}$. Las muestras fueron producidas mediante reacción de estado sólido. El análisis cristalográfico fue realizado a través de refinamiento Rietveld de los patrones experimentales de rayos X. Los resultados muestran que este material cristaliza en una perovskita ortorrómbica correspondiente al grupo espacial Pnma (\#62). Por medio del ajuste de Curie-Weiss a la respuesta de la susceptibilidad magnética en función de la temperatura se estableció que el ordenamiento magnético se relaciona con una transición paramagnético-antiferromagnético con una temperatura de Weiss $\theta=-18,5 \mathrm{~K}$, la cual es acorde con el comportamiento del inverso de la susceptibilidad en función de la temperatura, y una temperatura de Néel $\mathrm{T}_{\mathrm{N}}=50,8 \mathrm{~K}$. La constante de Curie permitió determinar un momento magnético efectivo de $15,7 \mu_{\mathrm{B}}$. Medidas de magnetización en función del campo aplicado a $\mathrm{T}=50 \mathrm{~K}$, muestran un débil comportamiento histerético, que corrobora el ordenamiento magnético presente a esa temperatura. Mediciones de la constante dieléctrica en función de la frecuencia aplicada a temperatura ambiente dan como resultado una alta constante dieléctrica relativa a bajas frecuencias $(\varepsilon=780)$. La curva de reflectancia en función de la longitud de onda revela el comportamiento típico de un material de tipo perovskita doble y permite la obtención de la brecha de energía de 2,74 eV característico de un material semiconductor.

a Grupo de Física de Nuevos Materiales, Universidad Nacional de Colombia, AA 5997, Bogotá, D.C., Colombia b Grupo Física de Materiales, Escuela de Física, Universidad Pedagógica y Tecnológica de Colombia, Tunja, Colombia

*jroar@unal.edu.co 


\begin{abstract}
We report structural analysis, surface morphology, magnetic ordering, dielectric response, optical feature and the electronic structure of the $\mathrm{Dy}_{2} \mathrm{BiFeO}_{6}$ novel complex perovskite. The samples were produced by the standard solid-state reaction recipe. Crystallographic analysis was performed by Rietveld refinement of experimental X-ray diffraction patterns. Results show that this material crystallizes in a perovskite with orthorhombic structure, which corresponds to the Pnma (\#62) space group. From the Curie-Weiss fitting on the curve of susceptibility as a function of temperature we establish that the ordering corresponds to a paramagnetic-antiferromagnetic transition, with a Weiss temperature $\theta=-18,5 \mathrm{~K}$, which is compatible with the behavior of the inverse of susceptibility as a function of temperature, and a Néel temperatura $\mathrm{T}_{\mathrm{N}}=50,8$ $\mathrm{K}$. The Curie constant allowed for us to obtain an effective magnetic moment of $15,7 \mu_{\mathrm{B}}$. The result of magnetization as a function of the applied field, measured at $\mathrm{T}=50 \mathrm{~K}$, shows a magnetic hysteresis behavior that corroborate the magnetic ordering present for this temperature value. Measurements of the dielectric constant as a function of applied frequencies at room temperature give as a result a high relative dielectric constant $(\varepsilon=780)$. The reflectance curve as a function of the wavelength reveals the typical behavior of a double perovskite-like material and permits to obtain the energy gap 2,74 eV, which is characteristic of a semiconductor material.
\end{abstract}

\section{INTRODUCCIÓN}

Las perovskitas son cerámicas que combinan elementos metálicos y no metálicos. La perovskita original $\mathrm{CaTiO}_{3}$ es un mineral escaso que cristaliza en forma ortorrómbica o seudocúbica [1], ya que se caracteriza porque su celda unitaria tiene forma geométrica con los tres ángulos rectos pero con parámetros de red distintos y es descrita por la expresión $\mathrm{ABO}_{3}$, donde $\mathrm{A}$ y $\mathrm{B}$ son cationes metálicos y $\mathrm{O}$ representa el Oxígeno [2]. Una vez producidas las llamadas perovskitas sintéticas, este nombre se generalizó a un grupo de cristales que comparten su misma disposición cristalográfica, con variaciones estructurales que van desde cúbica, tetragonal, ortorrómbica y monoclínica hasta romboédrica, por lo cual, dependiendo también de las propiedades físicas individuales de los cationes A y $\mathrm{B}$, pueden originarse comportamientos aislantes [3], semiconductores [4], conductores metálicos [5], magnetorresistivos [6], ferroeléctricos [7], ferromagnéticos [8], espin-metálicos [9] y superconductores [10], entre otros, lográndose la síntesis de estructuras cada vez más complejas como las perovskitas dobles, identificadas por la fórmula genérica $\mathrm{A}_{2} \mathrm{BB}^{\prime} \mathrm{O}_{6}$, donde $\mathrm{B}$ y $\mathrm{B}$ ' son generalmente metales de transición [11]. En los últimos años el interés por generar nuevos materiales de esta singular familia ha crecido debido a la posibilidad de mezclar adecuadamente las propiedades físicas de diversos elementos en los sitios estructurales A, B y B' de las perovskitas dobles, dando origen a compuestos con propiedades aplicables en tecnologías de nanomagnetismo y espintrónica [12]. En particular, la comunidad científica está interesada en la obtención de nuevos materiales que evidencien propiedades aplicables en razón de sus propiedades eléctricas y magnéticas [13], debido a sus múltiples aplicaciones en dispositivos para almacenamiento de información, válvulas de espín, sensores magnéticos y cabezas lectoras magnéticas, entre otros [14]. Un material bastante estudiado debido a sus propiedades multiferróicas es el $\mathrm{DyBiO}_{3}$, que es ferromagnética en temperaturas entre $50 \mathrm{~K}$ y $645 \mathrm{~K}$ además de ferroeléctrica en presencia de campos magnéticos aplicados a temperatura ambiente por causa de interacciones exóticas entre los orbitales 4f del Dy y 3d del Fe [15]. En la búsqueda de un nuevo material con propiedades físicas tecnológicamente útiles, en el presente trabajo se reportan algunas predicciones sobre las propiedades físicas del nuevo material $\mathrm{Dy}_{2} \mathrm{Bi}$ $\mathrm{FeO}_{6}$, que tiene como base la perovskita simple $\mathrm{DyFeO}_{3}$ con inclusión al $50 \%$ de $\mathrm{Bi}$ en el sitio del $\mathrm{Fe}$, con lo cual se espera una mejora en las propiedades dieléctricas del material. En el trabajo se detalla el proceso de síntesis y se presentan las caracterizaciones estructural, morfológica, óptica y magnética, estableciendo las correlaciones respectivas entre los resultados obtenidos para las diferentes técnicas experimentales. 


\section{PROCEDIMIENTO EXPERIMENTAL}

Muestras volumétricas de $\mathrm{Dy}_{2} \mathrm{BiFeO}_{6}$ fueron producidas mediante el método estándar conocida como reacción de estado sólido [4]. Los cálculos en proporciones estequiométricas para la producción de una muestra de un gramo de masa fueron 0,6828 g de $\mathrm{Dy}_{2} \mathrm{O}_{3}$ (Aldrich 99.99\%), 0,2420 g de $\mathrm{Bi}_{2} \mathrm{O}_{3}$ (Aldrich $99.9 \%$ ) y $0,0752 \mathrm{~g}$ de $\mathrm{Fe}_{3} \mathrm{O}_{4}$ (Aldrich 99.9\%), los cuales fueron secados a una temperatura de $100{ }^{\circ} \mathrm{C}$ durante dos horas y luego mezclados y molidos en un mortero de ágata durante 3 horas. Posteriormente, la mezcla en polvo obtenida se compactó para darle forma de pastilla cilíndrica y se sometió a un proceso de calcinación a $750 \circ \mathrm{\circ}$ durante 24 horas. Finalmente, la sinterización del material se realizó a $775{ }^{\circ} \mathrm{C}$ durante 24 horas, con lo cual se obtuvo la perovskita doble esperada, conforme se podrá constatar más adelante. La caracterización estructural se realizó por medio de la técnica de difracción de rayos $X$ en un equipo Panalytical X'PERT PRO, en la configuración geométrica Bragg-Brentano, en un rango angular $2 \theta$ entre $10^{\circ}$ y $90^{\circ}$, bajo la incidencia de una radiación de longitud de onda $\mathrm{CuK} \alpha$ de 1,540598 $\AA$ y una condiciones de trabajo de la fuente de $45 \mathrm{kV}$ y $40 \mathrm{~mA}$. El análisis de los datos experimentales tuvo lugar aplicando refinamiento tipo Rietveld con el software X'PERT Highscore, con el cual se obtuvieron directamente los parámetros estructurales y el grupo espacial correspondiente. Con el objeto de examinar la topografía superficial de las muestras, se aplicó la técnica de microscopía electrónica de barrido (MEB) mediante la utilización de un equipo VEGA 3 TESCAN con una resolución de $3 \mathrm{~nm}$ a $30 \mathrm{kV}$ en un vacío de $9 \times 10^{-3} \mathrm{~Pa}$. El análisis semicuantitativo que permitió corroborar la estequiometría del material fue efectuado usando la microsonda acoplada al microscopio, que permite la obtención de espectros dispersión de rayos X por electrones. Con el propósito de estudiar la respuesta magnética, se efectuaron medidas de susceptibilidad magnética en un equipo VersaLab Quantum Design, siguiendo los procedimientos ZFC (Zero Field Cooling) y FC (Field Cooled), en un rango de temperaturas entre $50 \mathrm{~K}$ y $300 \mathrm{~K}$, así como mediciones de magnetización en campos magnéticos variables hasta $\mathrm{H}=3 \mathrm{~T}$. La constante dieléctrica se determinó a través de mediciones de la constante relativa en función de la frecuencia para lo cual se usó un analizador de frecuencias HP8590. La caracterización óptica se efectuó por medio de un espectrofotómetro de reflectancia difusa Varian UV-Vis-NIR fabricado por Agilent Technologies. El análisis de los espectros se efectuó mediante ajustes siguiendo la teoría de Kubelka-Munk [16].

\section{RESULTADOS Y DISCUSIÓN}

El patrón de difracción resultante, debidamente refinado, para el material $\mathrm{Dy}_{2} \mathrm{BiFeO}_{6}$, es ejemplificado en la figura 1. En la misma, los símbolos representan los datos experimentales, la línea roja está asociada al difractograma simulado, y la línea azul es la diferencia entre los patrones teórico y experimental. En la figura 1 los planos de difracción respectivos aparecen marcados sobre los picos del patrón. Los parámetros de celda obtenidos a partir del refinamiento son presentados en la tabla 1 , donde $a, b, c$ representan los parámetros de red; $x, y, z$ las posiciones atómicas de los iones en la celda unitaria; $\alpha, \beta$, $\gamma$ son los ángulos entre las coordenadas en la celda; $\mathrm{X}^{2}, \mathrm{R}\left(\mathrm{F}^{2}\right) ; \mathrm{R}_{\mathrm{p}}$ y $\mathrm{R}_{\mathrm{wp}}$ son los parámetros de confiabilidad, los cuales dan información acerca de la calidad del procedimiento de refinamiento.

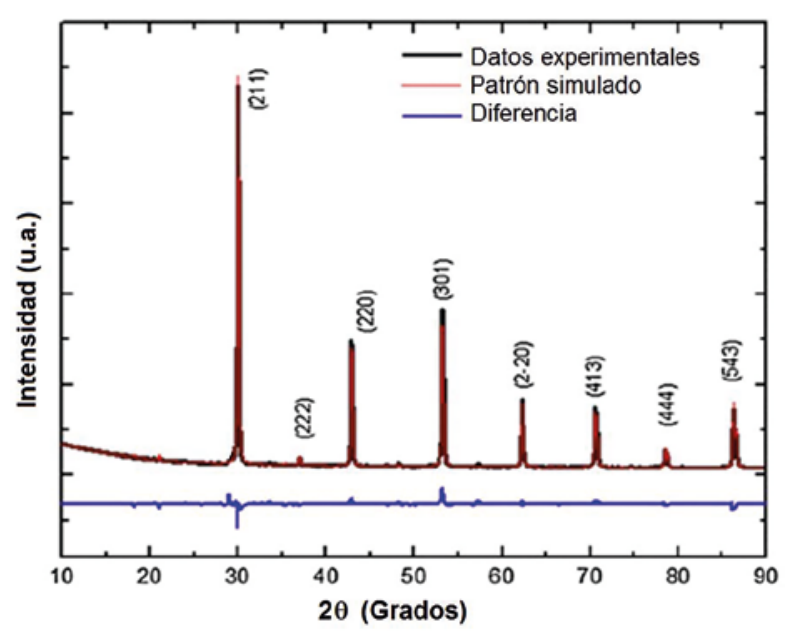

Figura 1. Difractograma refinado correspondiente a la perovskita doble $\mathrm{Dy}_{2} \mathrm{BiFeO}_{6}$. A partir del análisis se puede asegurar que el compuesto $\mathrm{Dy}_{2} \mathrm{BiFeO}_{6}$ cristaliza en una perovskita con estructura ortorrómbica y grupo espacial Pnma (\#62), con parámetros $b>a, c$, como se observa en la figura 2 . 
Tabla 1. Parámetros estructurales del material obtenido a partir del refinamiento Rietveld de los datos experimentales de difracción de rayos X.

\begin{tabular}{|c|c|c|c|c|c|}
\hline Grupo espacial & & $\# 62$ & Pnma & & \\
\hline Parámetros de red $(\stackrel{\circ}{A})$ & $a=5,596$ & $b=7,629$ & $c=5,301$ & $\alpha=\beta=\gamma=90^{\circ}$ & \\
\hline Elemento & $x$ & $y$ & $z$ & Ocupación & Wyckoff \\
\hline Dy & 0.0578 & 0.2500 & 0.4857 & 1.0 & $4 \mathrm{c}$ \\
\hline $\mathrm{Bi}$ & 0.0000 & 0.0000 & 0.0000 & 0.5 & $4 \mathrm{a}$ \\
\hline $\mathrm{Fe}$ & 0.0000 & 0.0000 & 0.0000 & 0.5 & $4 \mathrm{a}$ \\
\hline $\mathrm{O}$ & 0.4626 & 0.2500 & 0.6081 & 1.0 & $4 \mathrm{c}$ \\
\hline $\mathrm{O}$ & 0.3033 & 0.5557 & 0.1925 & 1.0 & $8 \mathrm{~d}$ \\
\hline
\end{tabular}

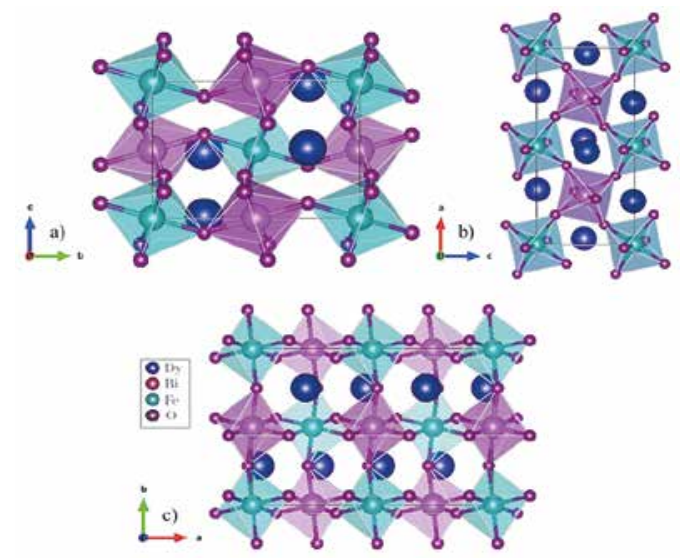

Figura 2. Perspectivas de la estructura a lo largo de los ejes cristalográficos $a, b$ y $c$.
La estructura ortorrómbica correspondiente es caracterizada por la notación de Glazer $a^{-} b^{+} a^{-}$ [17], para la cual dos ángulos de rotación con magnitudes muy similares y direcciones opuestas corresponden a un incremento de $\sqrt{2}$ en el parámetro de celda $a=c$, y doble valor del parámetro $b$ al compararla con una perovskita cúbica ideal Pmm. En otras palabras, el sistema de inclinación de Glazer sugiere la aparición de un tamaño de celda relativo a una celda unitaria de tipo cúbico con parámetro de red $a_{p}$, de tal manera que los parámetros en términos de $a_{p}$ están dados por $a=c=\sqrt{2} a_{p}$ y $b=2 a_{p}$. Con el fin de describir adecuadamente la estructura cristalográfica, se usa el concepto de las posiciones de Wyckoff, las cuales se presentan también en la tabla 2 [18].

Tabla 2. Posiciones de Wyckoff y parámetros estructurales del material $\mathrm{Dy}_{2} \mathrm{BiFeO}_{6}$.

\begin{tabular}{cccccc}
\hline Grupo espacial & \multicolumn{5}{c}{ Pnma } \\
\hline Parámetros de red $(\AA)$ & $a=5,596$ & $b=7,629$ & $c=5,301$ & $\alpha=\beta=\gamma=90^{\circ}$ & \\
\hline Elemento & $x$ & $y$ & $z$ & Ocupación & Wyckoff \\
\hline \hline Dy & 0.0578 & 0.2500 & 0.4857 & 1.0 & $4 \mathrm{c}$ \\
$\mathrm{Bi}$ & 0.0000 & 0.0000 & 0.0000 & 0.5 & $4 \mathrm{a}$ \\
$\mathrm{Fe}$ & 0.0000 & 0.0000 & 0.0000 & 0.5 & $4 \mathrm{a}$ \\
$\mathrm{O}$ & 0.4626 & 0.2500 & 0.6081 & 1.0 & $4 \mathrm{c}$ \\
$\mathrm{O}$ & 0.3033 & 0.5557 & 0.1925 & 1.0 & $8 \mathrm{~d}$ \\
\hline \hline
\end{tabular}

Las posiciones de Wyckoff determinan los sitios de los aniones y los cationes en la celda a través de una notación dada por letras que, en nuestro caso, son a, c y d, las cuales establecen todos los puntos $\mathrm{x}$ correspondientes a los grupos de simetría del sitio de la celda que son subgrupos del grupo Pnma [19]. Estas letras constituyen solo un marco de codificación para las posiciones de Wyckoff, comenzando con una cierta posición y continuando en orden alfabético. El número de puntos equivalentes por celda unidad, que acompaña a la letra de Wyckoff, se conoce como multiplicidad de la posición de Wyckoff [20]. 
En esta estructura, ocho átomos de oxígeno se sitúan en el plano $a c$, correspondiente a las posiciones de Wyckoff $8 d$, y otros cuatro átomos de oxígeno a lo largo del eje $b$ en las posiciones de Wyckoff $4 c$. Esta división del oxígeno en dos diferentes orbitales de Wyckoff constituye una evidencia de la aparición de una transición estructural desde una fase cúbica Pmm a una ortorómbica Pnma, ya que en una fase cúbica una sola posición de Wyckoff es suficiente para describir el oxígeno en la estructura. Por otro lado, a partir de la notación de Glazer $\left(a^{-} b^{+} a\right)$ es claro que se esperan distorsiones octaédricas con inclinaciones en antifase a lo largo de los ejes $a$ y $c$, e inclinación en fase a lo largo del eje $b$, donde $a$ y $b$ representan las longitudes de la celda pseudocúbica de Glazer [17].

Estas distorsiones octaédricas se pueden observar en las figuras 2 a y 2 c. En razón de lo anterior, los octaedros no están perfectamente alineados a lo largo de los ejes cristalográficos, presentando rotaciones e inclinaciones alrededor de estos ejes, tal que las distancias de los enlaces entre los cationes $\mathrm{Bi}^{3+}$ y $\mathrm{Fe}^{3+}$ y los aniones $\mathrm{O}^{2-}$ son diferentes a lo largo de los tres sub-ejes de cada octaedro. El análisis superficial de las muestras, efectuado a través de micrografías de MEB, es mostrado en la figura 3.

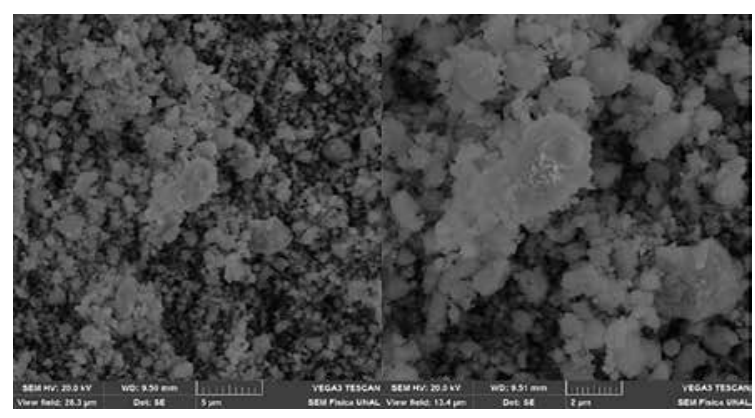

Figura 3. Imágenes de miscroscopía electrónica de barrido para las muestras de $\mathrm{Dy}_{2} \mathrm{BiFeO}_{6}$.

A partir de las imágenes de la figura 3 se puede establecer que las partículas del material poseen tamaños variados pero la mayoría de ellas no su- pera $2 \mu \mathrm{m}$. Adicionalmente puede observarse la formación de algunos clústers conformados por material particulado sub-micrométrico. Esta inhomogeneidad granular puede atribuirse a las bajas temperaturas de síntesis, las cuales fueron necesarias debido a la presencia del precursor trióxido de dibismuto, que posee temperatura de fusión muy baja $\left(822^{\circ} \mathrm{C}\right)$ [21]. Un análisis estadístico del tamaño de grano mediante el método de los interceptos dio como resultado un promedio de $1,25 \mu \mathrm{m}$, conforme se observa en la figura 4 .

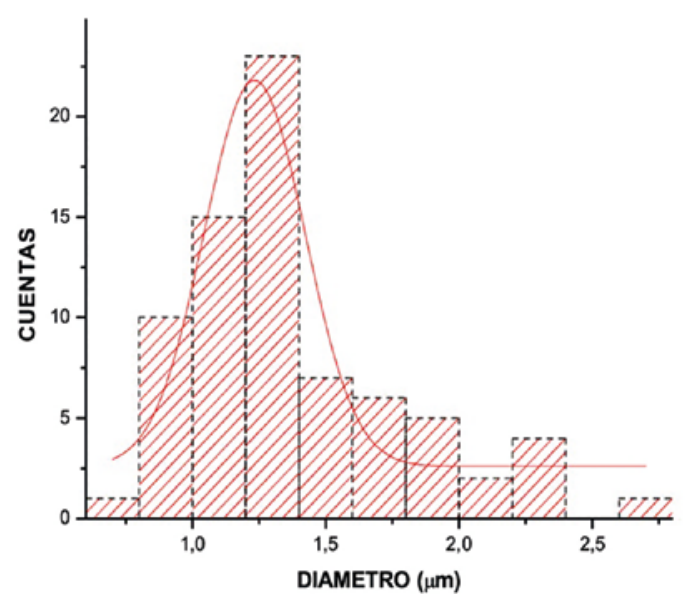

Figura 4. Determinación del tamaño de grano medio de los granos del material $\mathrm{Dy}_{2} \mathrm{BiFeO}_{6}$.

El análisis semicuantitativo por medio de espectroscopía de dispersión de rayos $\mathrm{X}$ por electrones es presentado en la figura 5. En el mismo es posible establecer el material no contiene otros componentes aparte de los esperados (Dy, Bi, Fe y O). A partir del área bajo la curva del espectro es posible determinar los porcentajes en peso de cada uno de los elementos constitutivos del material y compararlo con los porcentajes esperados a partir de la estequiometría del material, conforme se presenta en la tabla 3. Los resultados revelan que el material contiene la composición esperada hasta en un $98,1 \%$, lo cual es suficiente para concluir que se tiene la perovskita $\mathrm{Dy}_{2} \mathrm{BiFeO}_{6}$ en fase prácticamente pura, teniendo en cuenta que la técnica no es considerada cuantitativa debido a su dificultad para resolver los elementos livianos como el oxígeno. 


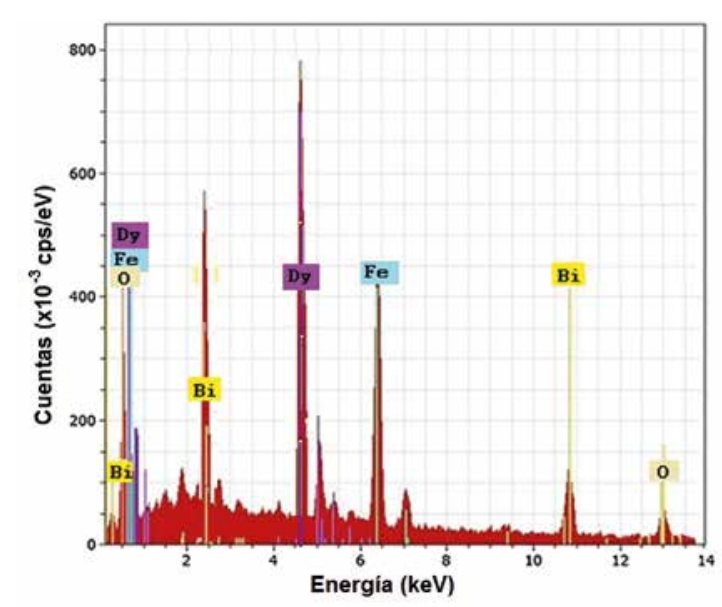

Figura 5. Espectro de dispersión de rayos $\mathrm{X}$ por electrones para la perovskita doble $\mathrm{Dy}_{2} \mathrm{BiFeO}_{6}$.

Tabla 3. Composición en porcentajes de masa teórico y experimental para el material $\mathrm{Dy}_{2} \mathrm{BiFeO}_{6}$.

\begin{tabular}{|c|c|c|}
\hline Elemento & \% Teórico & \% Experimental \\
\hline $\mathrm{Dy}$ & 47,39 & 48,29 \\
\hline $\mathrm{Bi}$ & 30,47 & 36,56 \\
\hline $\mathrm{Fe}$ & 8,14 & 8,29 \\
\hline $\mathrm{O}$ & 14,00 & 6,86 \\
\hline
\end{tabular}

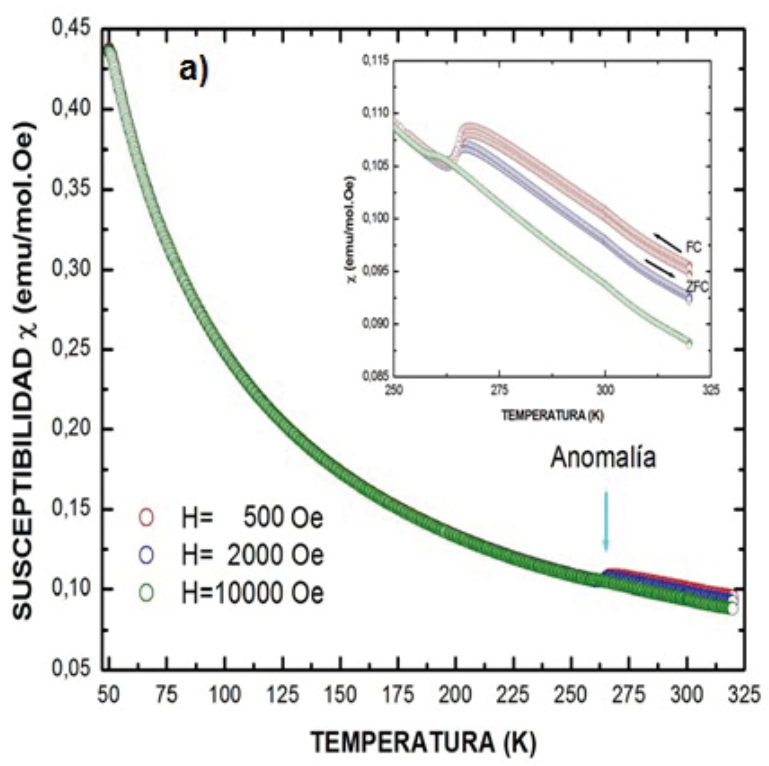

Las curvas de susceptibilidad magnética en función de la temperatura son mostradas en la figura 6a, en las cuales se aplicaron los procedimientos ZFC (enfriando en ausencia de campo magnético, aplicando el campo a bajas temperaturas y midiendo mientras la temperatura aumenta) y FC (midiendo mientras se enfría en presencia de campos magnéticos) bajo la aplicación de $\mathrm{H}=500,2000$ y 10000 Oe. El comportamiento observado evidencia una característica típicamente paramagnética, con un decrecimiento abrupto a bajas temperaturas y asintótico a altas temperaturas. Las unidades del momento magnético en el eje vertical emu tienen que ver con el acrónimo de "electromagnetic cgs units" y representan la cantidad de espines magnéticos orientados por el campo magnético aplicado. Esta cantidad es mayor a bajas temperaturas debido a que la entropía del sistema es menor, facilitando el efecto del campo magnético aplicado al sistema, de modo que el momento magnético tiende a variar con el inverso de la temperatura, conforme lo reportó Pierre Curie en 1895.

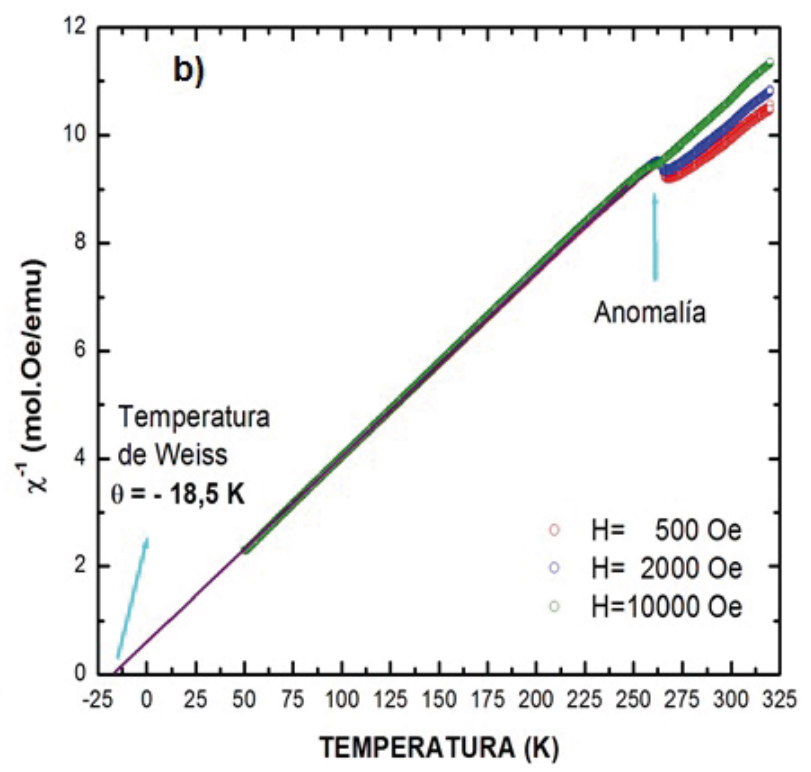

Figura 6. a) Comportamiento de la susceptibilidad en función de la temperatura bajo la aplicación de tres diferentes campos magnéticos; b) inverso de la susceptibilidad en función de la temperatura que facilita la obtención de la constante de Curie.

Al determinar la susceptibilidad magnética, multiplicando el momento magnético por la masa de la fórmula en $\mathrm{g} / \mathrm{mol}$ y dividiendo por la cantidad de masa de la muestra en $g$ y por el valor de intensidad de campo magnético en $O e$, las tres curvas escalan en una única curva, confor- me se esperaría, ya que en este tipo de materiales la magnetización y la susceptibilidad varían por un factor: el campo magnético aplicado, $\mathrm{M}=\chi \mathrm{H}$. De este modo se obtienen las curvas presentadas en la figura $6 \mathrm{a}$, en la que la pequeña diferencia ocurre entre las curvas ZFC y FC. Este compor- 
tamiento puede estar asociado a efectos de anisotropía de forma debido al carácter granular del material, ya que no todos los granos tienen la misma forma, con lo cual puede afectarse el acoplamiento magnético entre ellos, haciendo que los espines magnéticos respondan de manera diferente a la aplicación del campo magnético aplicado entre los diferentes granos de diversos tamaños y formas, resultando en un pequeño aumento de la susceptibilidad pero también en un efecto de desorden que genera irreversibilidad entre los dos procedimientos de medida (ZFC y FC). En la figura, las curvas de susceptibilidad para los tres campos magnéticos aplicados es esencialmente la misma, salvo por una pequeña anomalía observable para un valor de temperatura de $262,4 \mathrm{~K}$, a partir de la cual las curvas se separan ligeramente. Este efecto es más pronunciado cuanto menor es el campo magnético aplicado, conforme se puede ver en el recuadro de la figura 6a y puede deberse a la ocurrencia de efectos de anisotropía de forma relacionada con el carácter granular del material, conforme se evidenció en las micrografías anteriormente analizadas. Con el fin de establecer el tipo de respuesta magnética y teniendo en cuenta la predicción de Pierre Curie para materiales paramagnéticos, según la cual $\chi=$ $C / T$, donde $C=N \mu_{e f} 2 / 3 k_{B}$ es la constante de Curie, siendo $N$ el número de Avogadro, $\mu_{e f}$ el momento magnético efectivo del material y $k_{B}$ la constante de Boltzmann, se elaboró una gráfica del inverso de la susceptibilidad como se muestra en la figura 6 b. En la misma se observa claramente que la curva varía con el inverso de la temperatura, conforme lo predice la ley de Curie. Sin embargo, la extrapolación de la recta en el inverso de la susceptibilidad no corta el eje de la temperatura en el origen, por lo cual se establece que el material no se comporta como un paramagneto. Este tipo de comportamiento es típico de los materiales antiferromagnéticos, con una respuesta dada mediante la expresión $\chi=C /(T+\theta)$, conocida como ley de Curie-Weiss, donde $\theta=-18,5 \mathrm{~K}$ representa la temperatura de Weiss, que resulta negativa en este tipo de materiales, al contrario de los ferromagnéticos. A partir de la pendiente de la recta se determina el inverso de la constante de Curie, de donde se obtiene $C=30,22 \mu_{B} . \mathrm{K} / \mathrm{mol}$, siendo $\mu_{B}$ el magnetón de Bohr el momento magnético esperado para el espín de un único electrón aislado. De aquí se obtiene finalmente que el momento magnético efectivo del material es $\mu_{e f}=15,7 \mu_{B}$. Este valor corresponde a $96,3 \%$ del valor teórico esperado para el $\mathrm{Dy}_{2} \mathrm{BiFeO}_{6}$, el cual está dado por $\mu_{g}=\sqrt{2 \mu_{D y}^{2}+\mu_{B}^{2}+\mu_{\mathrm{Fe}}^{2}+6 \mu_{\mathrm{O}}^{2}} \approx 16,3 \mu_{B}$ donde se consideran proporcionalmente las contribuciones de todos los iones presentes en el material. La temperatura de Néel que caracteriza al material antiferromagnético, y que juega el mismo papel que la temperatura de Curie en el caso ferromagnético, debe encontrarse muy próxima y por encima de $\mathrm{T}=50 \mathrm{~K}$. Esta conclusión es inferida a partir de la pequeña curvatura observada en las curvas de susceptibilidad en función de la temperatura en $\mathrm{T}=50 \mathrm{~K}$, donde el comportamiento podría cambiar y empezar a disminuir con el decrecimiento de la temperatura, conforme se espera en este tipo de ordenamiento magnético. Otro indicio de la proximidad de la temperatura de Néel a los $50 \mathrm{~K}$ tiene que ver con la ocurrencia de una pequeña histéresis en las curvas de magnetización en función del campo magnético aplicado, medido a $\mathrm{T}=50 \mathrm{~K}$, como se observa en la figura $7 \mathrm{y}$ en su recuadro. Este comportamiento histerético se debe a la presencia de dominios magnéticos respondiendo a la orientación e intensidad de un campo magnético externo, ya que en el valor de la temperatura de Néel el material deja de ser paramagnético y, en ese punto, puede verse como un material ferromagnético, aunque se espera que por debajo de este valor de temperatura se formen dominios con momentos magnéticos orientados antiparalelos a la dirección del campo aplicado, anulando cualquier magnetización remanente en $\mathrm{T}=0 \mathrm{~K}$, ya que el material es antiferromagnético. A este valor de temperatura se determinó un campo coercitivo de 350 Oe y una magnetización remanente de $0,013 \mathrm{emu}$. Por otro lado, conforme se observa en la figura 7, a temperaturas $\mathrm{T}=200$ $\mathrm{K}$ y $\mathrm{T}=300 \mathrm{~K}$, claramente superiores a la temperatura de Néel, que es en este caso la temperatura de ordenamiento magnético, no se observa curva de histéresis debido a que el comportamiento es paramagnético puro. 


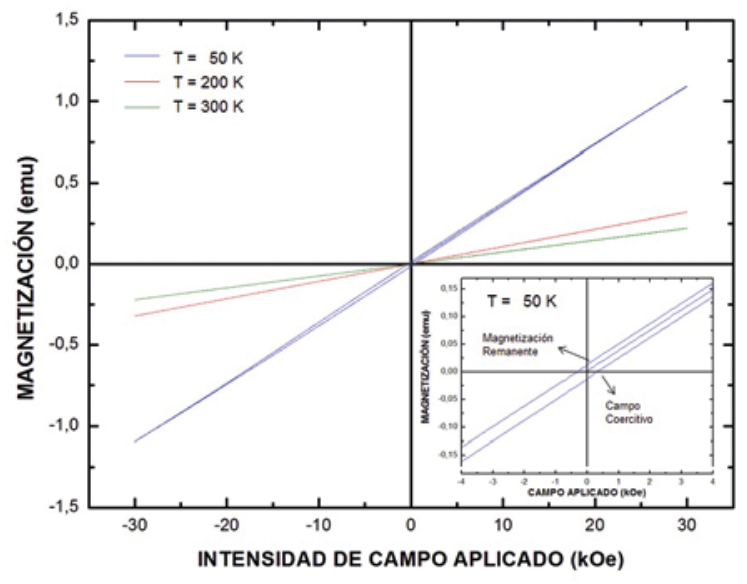

Figura 7. Curva de histéresis magnética en mediciones de magnetización en función del campo magnético aplicado.

La permitividad eléctrica relativa, también llamada constante dieléctrica, es una propiedad de los materiales que afecta las interacciones coulombianas entre dos puntos cargados eléctricamente en el material. Aunque hay varias formas de definirla, una de las más usuales es la razón entre las capacitancias de un material con dieléctrico y en el vacío. Como parte de la caracterización de nuestra perovskita compleja, la constante dieléctrica ha sido medida en función de la frecuencia aplicada en un rango entre $100 \mathrm{~Hz}$ y $40 \mathrm{MHz}$, conforme se muestra en la figura 8, en la cual puede verse que a bajas frecuencias este material evidencia una alta constante dieléctrica, la cual decae abruptamente entre 100 y $500 \mathrm{~Hz}$, permaneciendo en valores muy bajos, próximos de cero, para frecuencias mayores y por fuera de este régimen.

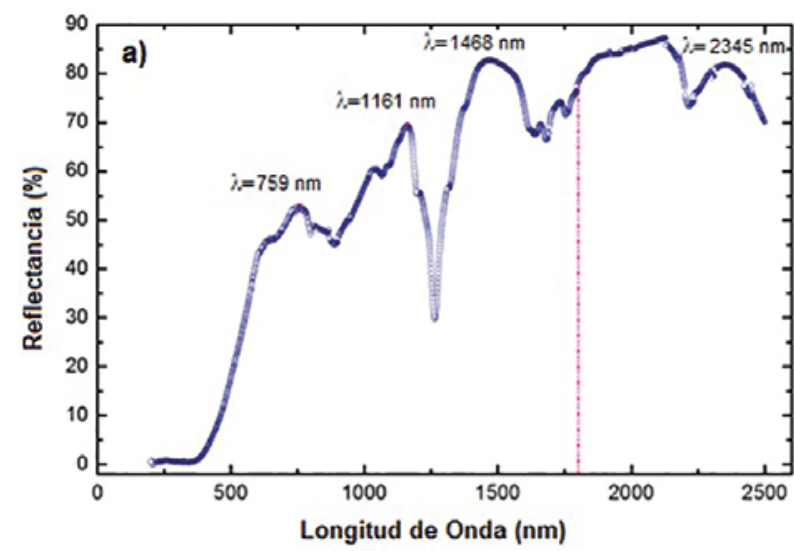

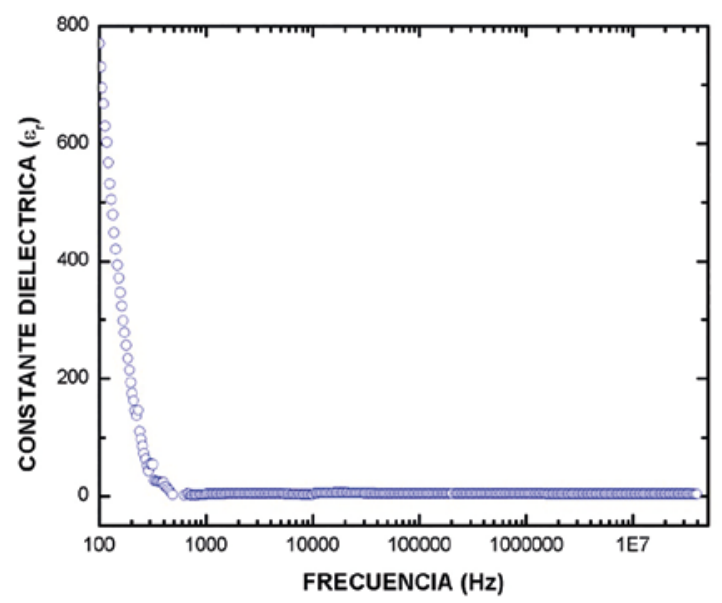

Figura 8. Constante dieléctrica relativa en función de la frecuencia para la perovskita compleja $\mathrm{Dy}_{2} \mathrm{BiFeO}_{6}$.

El valor de la constante dieléctrica obtenido a bajas frecuencias y temperatura ambiente $(\varepsilon \approx 780)$, resulta bastante mayor que el reportado para perovskitas de $\mathrm{SrTiO}_{3}(\varepsilon \approx 300)$ [22] y muy cercana al valor del material ferroeléctrico más estudiado $\mathrm{BaTiO}_{3}$, que a temperatura ambiente y bajas frecuencias presenta una constante dieléctrica $\varepsilon \approx 700-1000$ [23]. Por otro lado, resulta interesante que el valor de la brecha de energía reportado para el clásico $\mathrm{BaTiO}_{3}$ es 3,42 eV [24], muy próximo al que hemos obtenido experimentalmente para nuestra perovskita doble a partir de mediciones de Reflectancia Difusa. El alto valor de la constante dieléctrica sugiere eventuales aplicaciones de nuestro material, en condiciones estables de baja frecuencia y temperatura cercana a la temperatura ambiente, en dispositivos tales como capacitores cerámicos en multicapas (MLCs), termistores que evidencian resistencias con coeficiente térmico positivo (PTCR) y dispositivos microondas [25].

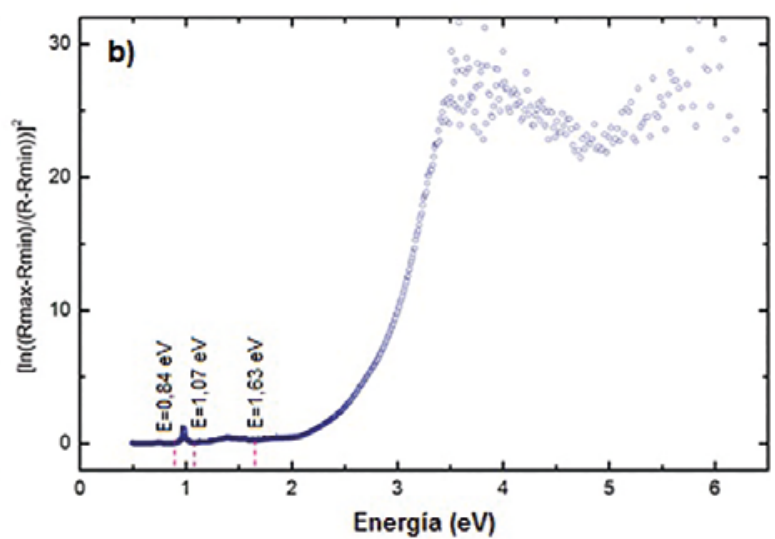

Figura 9. Respuesta óptica: a) espectro de reflectancia en función de la longitud de onda y b) en función de la energía. 
La caracterización óptica se efectuó a través de la técnica de reflectancia difusa, en la cual se obtiene el espectro de porcentaje de reflectancia de un sólido en función de la longitud de onda. Este espectro es presentado en la figura 9a para la perovskita doble $\mathrm{Dy}_{2} \mathrm{BiFeO}_{6}$, en la que se observa la ocurrencia de cuatro picos característicos. El primero, mostrado en $\lambda=2345 \mathrm{~nm}$, corresponde al régimen de bajas energías y está asociado a efectos de la técnica al detectar vibraciones de la celda completa y no de sus átomos independientemente. Los otros tres picos para las longitudes de onda $\lambda_{1}=759 \mathrm{~nm}, \lambda_{2}=1161 \mathrm{~nm}$ y $\lambda_{3}=1468 \mathrm{~nm}$, que equivalen a las energías $\mathrm{E}_{1}=1,63 \mathrm{eV}, \mathrm{E}_{2}=1,07$ $\mathrm{eV} \mathrm{y} \mathrm{E}_{3}=0,84 \mathrm{eV}$ respectivamente, son producto de las excitaciones debidas a vibraciones de los enlaces catión-anión dentro de la celda unitaria. La figura 9b ilustra la dependencia de la respuesta óptica con la energía.

Las bandas observadas en el espectro de reflectancia se deben a modos activos de fonones perceptibles en el Infrarrojo. Para el grupo espacial Pnma (\#62), el grupo puntual de simetría co-

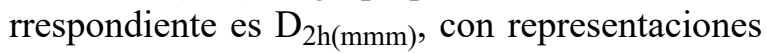
irreductibles que dependen del número de átomos de la celda unidad y de los grados de libertad de traslación, rotación y vibración. En nuestro caso, se espera una representación irreductible dada por una expresión que contiene cinco términos correspondientes a vibraciones observables en espectroscopía Raman y tres en Infrarrojo así: $\mathrm{T}=7 A_{g}^{R}+7 B_{1 g}^{R}+5 B_{1 g}^{R}+5 B_{2 g}^{R}+8 A_{v}^{R}+7 B_{1 u}^{l R}+9 B_{2 u}^{\prime R}+9 B_{3 u}^{l / F}$ donde los subíndices denotan los diferentes modos de vibración. En la anterior expresión el superíndice $\mathrm{R}$ representa modos de vibración debidos a energías absorbidas que coinciden con la diferencia energética entre niveles, convirtiendo la energía de los fotones que es absorbida, en energía fonónica en forma de vibraciones Raman. El superíndice IR se refieren a vibraciones de dipolos moleculares oscilantes debidos a absorciones por resonancia. En particular, los modos $\mathrm{B}_{1 \mathrm{u}}, \mathrm{B}_{2 \mathrm{u}}$ y $\mathrm{B}_{3 \mathrm{u}}$ se relacionan con las posiciones estructurales de Wyckoff representados en la tabla 4, donde los números que aparecen bajo los modos activos del Infrarrojo corresponden a los caracteres asociados a los elementos de simetría en la representación irreductible del grupo espacial Pnma. Estos modos activos constituyen momentos dipolares inducidos debido a vibraciones que producen cambios en las posiciones atómicas de la celda unidad. En el caso del $\mathrm{Dy}_{2} \mathrm{BiFeO}_{6}$, los modos vibracionales aparecen como picos de absorción en el espectro de reflectancia difusa de la figura 9a y se relacionan con vibraciones en los enlaces Dy-O, Bi-O y Fe-O. De lo anterior, puede inferirse que el pico de mayor frecuencia corresponde al enlace $\mathrm{Fe}-\mathrm{O}$, ya que en este la distancia interatómica es la menor. En este orden de ideas, el de menor frecuencia corresponde al enlace Dy-O y el de frecuencia intermedia al enlace Bi-O.

Tabla 4. Modos activos del Infrarrojo para las distintas posiciones de Wyckoff.

\begin{tabular}{clll}
\hline \multirow{2}{*}{ Posición de Wyckoff } & \multicolumn{3}{c}{ Modos Activos IR } \\
\cline { 2 - 4 } & $B_{1 u}$ & $B_{2 u}$ & $B_{3 u}$ \\
\hline \hline $4 \mathrm{a}$ & 3 & 3 & 3 \\
\hline $4 \mathrm{~b}$ & 3 & 3 & 3 \\
\hline $4 \mathrm{c}$ & 2 & 1 & 2 \\
\hline $8 \mathrm{~d}$ & 3 & 3 & 3 \\
\hline
\end{tabular}

La figura 10 muestra la representación de $[\ln [(\mathrm{Rmax}-\mathrm{Rmin}) /(\mathrm{R}-\mathrm{Rmin})]]^{2}$ en función de la energía, que es una corrección al modelo de $\mathrm{Ku}$ belka-Munk [17] que permite la obtención directa de la brecha de energía mediante extrapolación hacia el eje de la energía.

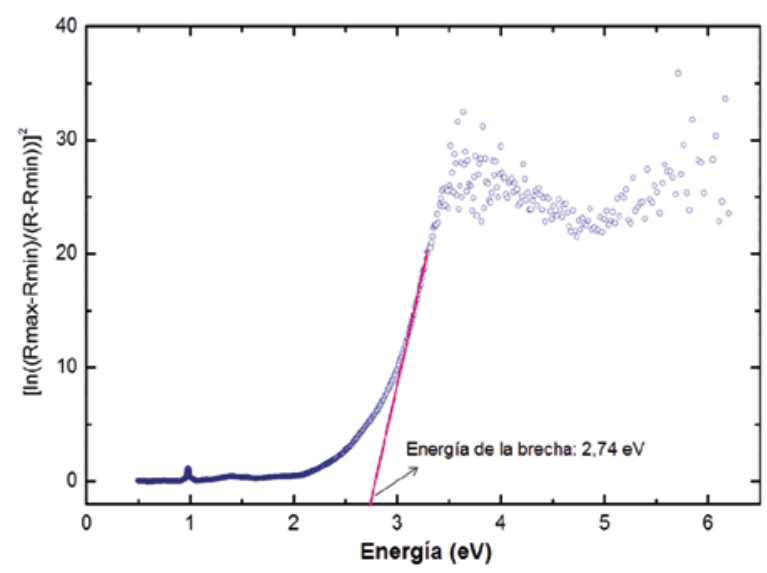

Figura 10. Determinación experimental de la brecha de energía.

A partir de este procedimiento se obtuvo un valor de brecha de energía de 2,74 eV, que permite caracterizar el material como un semiconductor. 


\section{CONCLUSIONES}

Los resultados del refinamiento de los datos experimentales de difracción de rayos $\mathrm{X}$ revelan que se ha logrado sintetizar el material Dy${ }_{2} \mathrm{BiFeO}_{6}$ en una fase ortorrómbica perteneciente al grupo espacial Pnma. Todos los parámetros cristalográficos fueron determinados a partir del análisis Rietveld, concluyéndose que la estructura presenta evidencias de distorsiones octahédricas en fase a lo largo del eje $b$ de la celda cristalina y en desfase a lo largo de los ejes $a$ y $c$. La caracterización morfológica superficial permitió observar la presencia de granos de tamaño inhomogéneo cuyo promedio se determinó en $2 \mu \mathrm{m}$, con la aparición de granos submicrométricos aglomerados en clústers de varios micrómetros de extensión. La microsonda de dispersión de rayos $\mathrm{X}$ por electrones reveló que en un $98,1 \%$ el contenido del material corresponde con el esperado a partir de la estequiometría esperada. La caracterización magnética del $\mathrm{Dy}_{2} \mathrm{BiFeO}_{6}$ permite concluir que el material tiene comportamiento paramagnético a temperatura ambiente, donde todos los espines responden independientemente a la aplicación de un campo magnético externo, y presenta una transición al estado antiferromagnético por debajo de la temperatura de Néel, que se presume próxima a $\mathrm{T}=50 \mathrm{~K}$, por debajo de la cual los momentos magnéticos adoptan una estructura de dominios con interacción de intercambio negativa, es decir, algunos se alinean en dirección del campo magnético aplicado y otros en dirección opuesta al campo externo. El momento magnético efectivo resulta aproximado al valor esperado en un $96,3 \%$, considerando una contribución mayoritaria de los espines de los electrones desapareados,

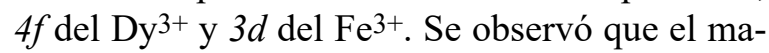
terial presenta una elevada respuesta dieléctrica a bajas frecuencias $(\varepsilon \approx 780)$, siendo del orden del esperado en materiales ferroeléctricos como el $\mathrm{BaTiO}_{3}$, con lo cual podría tener eventuales aplicaciones en dispositivos basados en microondas. Finalmente, la caracterización óptica mostró el carácter semiconductor del $\mathrm{Dy}_{2} \mathrm{BiFeO}_{6}$, con la ocurrencia de una brecha de energía de 2,74 eV. Por lo anterior, se puede concluir que la doble perovskita $\mathrm{Dy}_{2} \mathrm{BiFeO}_{6}$ se comporta como un buen dieléctrico a temperatura ambiente, donde el material es paramagnético, y adopta una naturaleza antiferromagnética a temperaturas próximas de $\mathrm{T}=50 \mathrm{~K}$.

\section{AGRADECIMIENTOS}

Los autores agradecemos a la DIB, Universidad Nacional de Colombia, Sede Bogotá, por el apoyo parcial y K.Y. Bustos Garnica agradece especialmente a Colciencias dentro del programa de Jóvenes Investigadores.

\section{REFERENCIAS}

[1] P. García-Fernández, J.A. Aramburu, M.T. Barriuso, M. Moreno: Key Role of Covalent Bonding in Octahedral Tilting in Perovskites, Journal of Physical Chemistry Letters 1 , 647-651 (2010).

[2] Hazen RM (1988) Perovskites. Scientific American 258, \#6, 74-81.

[3] Q. Madueño, D.A. Landínez Téllez, J. Roa-Rojas, Production and characterization of $\mathrm{Ba}_{2} \mathrm{NdSbO}_{6}$ complex perovskite as a substrate for $\mathrm{YBa}_{2} \mathrm{Cu}_{3} \mathrm{O}_{7-\delta}$ superconducting films, Modern Physics Letters B 20, 427 (2006).

[4] J. A. Cuervo Farfán, D. M. Aljure García, R. Cardona, J. Arbey Rodríguez, D.A. Landínez Téllez, J. Roa-Rojas, Structure, Ferromagnetic, Dielectric and Electronic Features of the LaBiFe2O6 Material, Journal of Low Temperature Physics Volume 186, Issue 5, pp 295-315 (2017).

[5] Y-Q. Zhai, J. Qiao, Z. Zhang, Magnetic and Electrical Transport Properties of Double Perovskite Sr2FeMoO6 Prepared by SolGel Method, E-Journal of Chemistry 2011, 8(S1), S189-S194.

[6] B. Raveau , A. Maignan , C. Martin , M. Hervieu, Colossal Magnetoresistance Manganite Perovskites: Relations between Crystal Chemistry and Properties, Chem. Mater., 1998, 10 (10), pp 2641-2652.

[7] DA Landinez et al DYNA 2013

[8] J Sánchez-Benítez, M J Martínez-Lope, J A Alonso, J L García-Muñoz, Magnetic and structural features of the NdNi1-xMnxO3 
perovskite series investigated by neutron diffraction, Journal of Physics: Condensed Matter, Volume 23, Number 22, 226001 , 2011.

[9] M Bonilla, DAL Téllez, JA Rodríguez, JA Aguiar, J Roa-Rojas, Study of half-metallic behavior in Sr 2 CoWO 6 perovskite by ab initio DFT calculations, Journal of Magnetism and Magnetic Materials 320 (14), e397-e399 (2008).

[10] Iván Supelano García, Armando Sarmiento Santos, Carlos Arturo Parra Vargas, David Landínez Téllez, Jairo Roa Rojas, "Síntesis y propiedades estructurales del sistema superconductor La1,5+xBa1,5+x-yCayCu3Oz" Ciencia En Desarrollo v.4 p.27 32, 2013.

[11] D.A. Landínez Téllez, C.E. Deluque Toro R. Cardona J. Roa-Rojas: Cristalographic, Ferroelectric and Electronic Properties of the Sr2ZrTiO6 Double Perovskite, Modern Physics Letters B 27(20):50141- August 2013.

[12] Bekir Aktaş, Faik Mikailzade, Bulat Rameev, Numan Akdoğan, Recent advances in nanomagnetism and spintronics J3M Volume 373, 1 January 2015, Pag 1.

[13] Y. Tokura, N. Kida, Dynamical magnetoelectric effects in multiferroic oxides, Phil. Trans. R. Soc. A (2011) 369, 3679-3694.

[14] M.M. Vopson, Fundamentals of Multiferroic Materials and Their Possible Applications, Journal Critical Reviews in Solid State and Materials Sciences 40(4), 223-250, 2015.

[15] B. Rajeswaran, D. Sanyal, Mahuya Chakrabarti, Y. Sundarayya, A. Sundaresan, C. N. R. Rao, Interplay of 4f-3d magnetism and ferroelectricity in $\mathrm{DyFeO}_{3}$, Europhys. Lett. 101(1), 17001 (2013).
[16] V. Kumar, S. Kr. Sharma, T.P. Sharma, V. Singh, Band gap determination in thick fil$\mathrm{ms}$ from reflectance measurements, Optical Materials 12 (1999) 115-119.

[17] C.J. Howard, H.T. Stokes, Group-Theoretical Analysis of Octahedral Tilting in Perovskites, Acta Cryst. (1998). B54, 782-789.

[18] C.A. Triana, D.A. Landínez Téllez, J. Roa-Rojas, General study on the crystal, electronic and band structures, the morphological characterization, and the magnetic properties of the $\mathrm{Sr}_{2} \mathrm{DyRuO}_{6}$ complex perovskite, Materials Characterization 99, 128141 (2015).

[19] L.A. Carrero Bermúdez, R. Moreno Mendoza, R. Cardona, D.A. Landínez Téllez, J. Roa-Rojas, Journal of Experimental and Theoretical Nanotechnology Specialized Researches 1, 197 (2017).

[20] W. Wondratschek, International Tables for Crystallography (2006), Vol. A, Chapter 8.3, pp. 732-740.

[21] F. Schröder, N. Bagdassarov, F. Ritter, L. Bayarjargal, (2010) Temperature dependence of $\mathrm{Bi}_{2} \mathrm{O}_{3}$ structural parameters close to the $\alpha-\delta$ phase transition, Phase Transitions, 83: $5,311-325$.

[22] B.K. Choudhuray, K.V. Rao, R.N.P. Choudhary, J. Mater. Sci. 24, 3469 (1989).

[23] P.R. Arya, P. Jha, G.N. Subbanna, A.K. Ganguli, Mater. Res. Bull. 38, 617 (2003).

[24] S. Piskunov, E. Heifets, R.I. Eglitis, G. Borstel, Computat. Mater. Sci. 29, 165 (2004).

[25] M.M. Vijatović, J.D. Bobić, B.D. Stojanović, Science of Sintering 40, 235 (2008). 